\title{
Reliability of Sound Field Simulations
}

\author{
J. WIERZBICKI* \\ AGH University of Science and Technology, Department of Mechanics and Vibroacoustics \\ al. Mickiewicza 30, 30-059 Kraków, Poland
}

\begin{abstract}
One of the most important questions in sound field modelling is connected with the reliability of results. The absolute accuracy of values predicted by the software is very difficult to quantify because of an uncertainty in the measurements. But it is possible to determine an influence of the input data accuracy and calculation parameters on the obtained results. Problems and differences in the sound field simulation results are presented in the hereby paper. Test procedures of the sound filed simulation software and also well-documented exemplary objects in an open space will be the main aim of investigations.
\end{abstract}

PACS numbers: 43.58.Ta, 43.55.Ka

\section{Introduction}

In the first round of noise mapping and created from them action plans only the results obtained from the calculation software are used. Because an economic impact of action plans is very significant there will be probably many reservations or objections regarding noise control decisions which should be very well documented. The most important question connected with noise mapping realized by computational methods concerns the reliability of results. It is closely related to the quality of input data and calculation algorithms and their parameters. A process of collecting input data for noise mapping is not only expensive but also difficult and time-consuming. Since some kinds of data are more important than the other, one can accelerate such process and reduce costs by classifying the data acquisition from the most to the least important. However, the difficulty is connected with the fact that the importance depends on the arrangement of elements and is different e.g. for open spaces and for urban areas.

The main aim of the research is to obtain an answer concerning the uncertainty of results received from the sound field simulation software. Such software uses ray methods (geometric models) for a sound field modelling and the accuracy of results strongly depends on the number of ray reflections which, in turn, is related to the number and shape of the modelled objects.

\section{Accuracy guidelines}

The Environmental Noise Directive (END) requires noise mapping results which are sufficiently accurate that dividing them into discrete $5 \mathrm{~dB}(\mathrm{~A})$ wide sets is an appropriate process. It means that the absolute accuracy should be within $2 \mathrm{~dB}(\mathrm{~A})$ of the actual value [1,2]. For

* corresponding author; e-mail: wierzbic@agh.edu.pl the recommended for the European Union members Harmonoise/Imagine reference model, an overall accuracy is defined for the full $L_{\mathrm{DEN}}$ assessment, which is half the accuracy as for the engineering model. The following accuracy, expressed in terms of the expected standard deviation in the predicted noise levels, should be reached for the reference model [3]:

- $\pm 1 \mathrm{~dB}$ for a free field and distances up to $100 \mathrm{~m}$,

- $\pm 2 \mathrm{~dB}$ for a flat terrain and distances up to $2000 \mathrm{~m}$,

- $\pm 5 \mathrm{~dB}$ for a mountainous terrain and built-up urban areas.

Such numbers "do not seem to be very ambitious values, but it is felt that this is already a big improvement compared to the existing models" [3].

\section{Sources of uncertainty}

Within any modelling system designed to reproduce a real world environment, such as a software for noise mapping, there are four sources of uncertainty to be considered [4]:

1. Input uncertainties - estimation of the uncertainty in model inputs and parameters (involves a study of each of the various types of data required to construct a noise map);

2. Uncertainty propagation - estimation of the uncertainty in model outputs resulting from the uncertainty in model inputs and model parameters;

3. Model uncertainty - uncertainty associated with different model structures and model formulations;

4. Uncertainty in model predictions resulting from the uncertainty in the evaluation data. 
Uncertainties of type 2 and 3 are considered in this paper.

A sound source or measurement point position indicated by the GPS can be a good example of "input uncertainty". Many tests with the commercial GPS equipment were performed and the obtained results were not completely repeatable. The same path between three points in an open space presented in Fig. 1 indicates differences of about $3-5 \mathrm{~m}$. The arbitrarily selected $3 \mathrm{~m}$ error is considered in this paper.

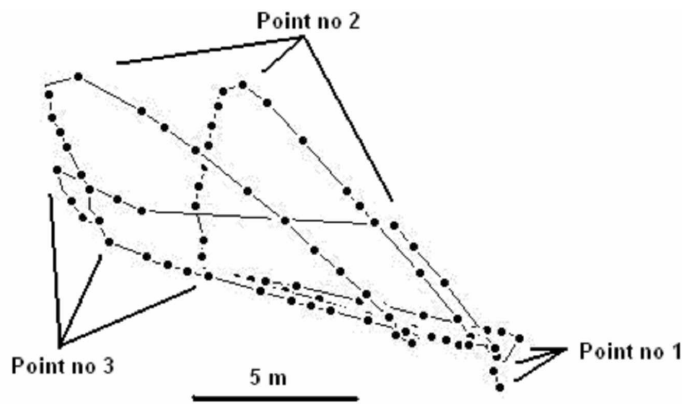

Fig. 1. Differences in GPS indicated positions - the same path between three points in an open space recorded at daily intervals.

\section{Experiments}

All experiments are connected with the estimation of type 2 and 3 uncertainties. When there are no input data available or they are limited, one should use Toolkits from [4], e.g. when there is no traffic flow data available default numbers of vehicles should be used in order to achieve accuracy in $4 \mathrm{~dB}$ margin - Table I. The accuracy presented in toolkits is not related to the distance between a sound source and a measurement point and buildings type (even for building heights - toolkit 15). The performed experiments can show if it is a correct assumption.

For example, one of the simplest test is presented in Fig. 2. It was assumed that a vehicle flow estimation was made with $\pm 20 \%$ error and the receiver position was specified with $\pm 3 \mathrm{~m}$ margin. Then the calculations were made for all combinations of flow and receiver distances for 3 receiver heights using two commercial programs (CadnaA and SoundPlan).

The results are presented in a tabular form for each experiment. It is clear that the biggest value is for the distance change of the nearest receiver but the results show that there are also differences between programs. They provide nearly the same values for $10 \mathrm{~m}$ and $100 \mathrm{~m}$ distances but for $2000 \mathrm{~m}$ the difference is outside the END limits - Table II.

\section{Results}

On the bases of several dozen performed experiments one can notice that:

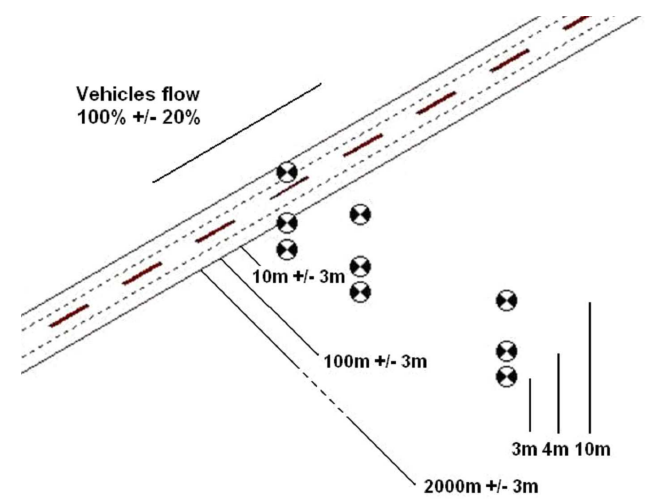

Fig. 2. Test of model multi-input uncertainty estimation (uncertainty propagation) - an influence of vehicle flow and distance from road with GPS accuracy on calculated values of $L_{\mathrm{DEN}}$ in selected points (black and white circles).

- Results from various software are different - the maximum difference is even more than $5 \mathrm{~dB}$. The accuracy of calculated results depends on the software user experience - differences can be caused e.g. by freely selected number of reflections.

- The calculation software should be improved especially in input data interface and calculation options (all input data should be tested e.g. buildings height - whether one is mistakenly much higher than others or has $0 \mathrm{~m}$ height - the user should be asked about it, a number of reflections should be selected by the software, grid sizes should vary and should be optimised and selected automatically by the software).

- An influence of an input data inaccuracy on the calculated values depends on the acoustic elements configuration (free field, urban area etc.). For example in some cases the reliable data about the road surface type are more important than buildings positions (Table III).

Such "Tables of significance" designed for typical configurations (e.g. a flat terrain without and with barriers, buildings near main roads) should shorten the preparation time of input data and the total calculation time.

\section{Conclusions}

- It seems that in the first round of noise mapping the calculation software should be used rather for calculating differences in $L_{\mathrm{DEN}}$ after modifications (e.g. barriers height, vehicles flow) than for accurate values of $L_{\mathrm{DEN}}$. The absolute accuracy (uncertainty) of the predicted by the software sound levels is very difficult to quantify because of the measurement uncertainty. 
TABLE I

TABLE II

Default number of vehicles when no traffic flow data are available [4].

\begin{tabular}{l|c|c|c}
\hline \hline \multicolumn{1}{c|}{ Road type } & Day & Evening & Night \\
\hline Dead-end roads & 175 & 50 & 25 \\
Service roads & 350 & 100 & 50 \\
Collecting roads & 700 & 200 & 100 \\
Small main roads & 1400 & 400 & 200
\end{tabular}

- It is necessary to inform about the uncertainty of the calculated values of $L_{\mathrm{DEN}}$ but there are several such values dependent on the type of modelled objects and terrain.

- It seems also that the monitoring system with long-term $L_{\mathrm{DEN}}$ calculations and sound sources recognition should be the obligatory part of noise mapping. Such solution is necessary not only for the calibration of prediction software but especially for the real-time noise mapping system.

Differences between calculated by two commercial programs values of $L_{\mathrm{DEN}}$.

\begin{tabular}{c|c|c|c|c}
\hline \hline \multirow{2}{*}{$\begin{array}{c}\text { Receiver } \\
\text { Height }\end{array}$} & \multirow{2}{*}{$\begin{array}{c}\text { Vehicles } \\
\text { Flow }\end{array}$} & \multicolumn{3}{|c}{ Distance from road } \\
& & $10 \mathrm{~m}$ & $100 \mathrm{~m}$ & $2000 \mathrm{~m}$ \\
\cline { 3 - 5 } $3 \mathrm{~m}$ & $80 \%$ & $0.3 \mathrm{~dB}$ & $0.1 \mathrm{~dB}$ & $\mathbf{3 . 4} \mathrm{dB}$ \\
& $100 \%$ & $0.2 \mathrm{~dB}$ & $0.1 \mathrm{~dB}$ & $3.3 \mathrm{~dB}$ \\
& $120 \%$ & $0.2 \mathrm{~dB}$ & $0 \mathrm{~dB}$ & $3.2 \mathrm{~dB}$ \\
\hline \multirow{3}{*}{$4 \mathrm{~m}$} & $80 \%$ & $0.2 \mathrm{~dB}$ & $0.1 \mathrm{~dB}$ & $\mathbf{3 . 4} \mathrm{dB}$ \\
& $100 \%$ & $0.2 \mathrm{~dB}$ & $0.1 \mathrm{~dB}$ & $3.3 \mathrm{~dB}$ \\
& $120 \%$ & $0.2 \mathrm{~dB}$ & $0 \mathrm{~dB}$ & $3.3 \mathrm{~dB}$ \\
\hline \multirow{3}{*}{$10 \mathrm{~m}$} & $80 \%$ & $0.2 \mathrm{~dB}$ & $0.2 \mathrm{~dB}$ & $3.3 \mathrm{~dB}$ \\
& $100 \%$ & $0.1 \mathrm{~dB}$ & $0.2 \mathrm{~dB}$ & $3.2 \mathrm{~dB}$ \\
& $120 \%$ & $0.1 \mathrm{~dB}$ & $0.2 \mathrm{~dB}$ & $3.3 \mathrm{~dB}$
\end{tabular}

\section{TABLE III}

"Table of significance" for urban area (buildings near main road) - possible accuracy in sound level calculations [4] related to variation of input data - geometry of objects (geom.), acoustic parameters (param.) and calculation algorithms (alg.) and maximal values of accuracy obtained from experiments.

\begin{tabular}{c|l|c|c|c|c|c}
\hline \hline \multicolumn{2}{c|}{ Significance of Parameter } & Parameter & \multicolumn{2}{|c|}{ Possible Accuracy [4] } & Max. Accur. \\
\cline { 3 - 6 } & Type & $<1 \mathrm{~dB}$ & $1-5 \mathrm{~dB}$ & $>5 \mathrm{~dB}$ & {$[\mathrm{~dB}]$} \\
\hline 1 & Sound sources position & geom. & $\mathrm{XXX}$ & $\mathrm{XXX}$ & $\mathrm{XXX}$ & 11.7 \\
2 & Number of reflections & alg. & $\mathrm{XXX}$ & $\mathrm{XXX}$ & $\mathrm{XXX}$ & 7.0 \\
3 & Buildings height & geom. & $\mathrm{X}$ & $\mathrm{X}$ & & 5.0 \\
4 & Road surface type & param. & $\mathrm{XXX}$ & $\mathrm{XXX}$ & $\mathrm{XXX}$ & 4.5 \\
5 & Calculation standard & alg. & $\mathrm{X}$ & $\mathrm{X}$ & & 3.9 \\
6 & Buildings positions & geom. & $\mathrm{X}$ & $\mathrm{X}$ & & 3.4 \\
7 & Number of vehicles & param. & $\mathrm{XXX}$ & $\mathrm{XXX}$ & $\mathrm{XXX}$ & 3.0 \\
8 & Road type & param. & $\mathrm{X}$ & $\mathrm{X}$ & & 2.6 \\
9 & Buildings cross-section sizes & geom. & $\mathrm{X}$ & $\mathrm{X}$ & & 2.4 \\
10 & Road width & geom. & & & & 0.2 \\
11 & Vegetation & param. & & & & 0 \\
12 & Number of residents & alg. & & & & 0
\end{tabular}

\section{References}

[1] Directive 2002/49/EC of the European Parliament and of the Council of 25 June 2002 relating to the assessment and management of environmental noise.

[2] The Department for Environment, Food and Rural Affairs (Defra), Research project NAWR 93: $W G-$ AEN's Good Practice Guide and the Implications for Acoustic Accuracy. (2005).
[3] Imagine Project. Available from http://www.imagine-project.org/ .

[4] European Commission Working Group Assessment of Exposure to Noise (WG-AEN) Position Paper Good Practice Guide for Strategic Noise Mapping and the Production of Associated Data on Noise Exposure Version 2. (2006). 